\title{
Análise da influência do exercício físico em idosos com relação a equilíbrio, marcha e atividade de vida diária
}

Analysis of the influence of physical exercise on balance, motion and activity of daily living in elderly people

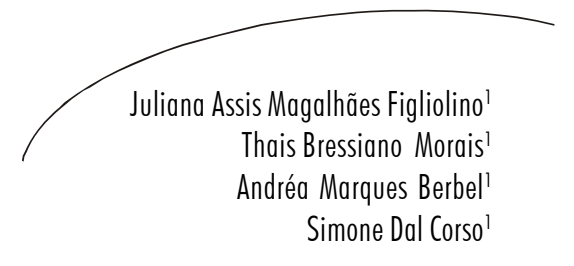

\section{Resumo}

Objetivo: Avaliar a influência do nível de atividade física no perfil lipO objetivo foi comparar idosos praticantes e não praticantes de atividade física, em relação à probabilidade de quedas, desequilíbrio e dificuldades na realização das atividades de vida diária (AVD). Trata-se de um estudo transversal com a participação de 40 idosos, de ambos os gêneros. Os idosos foram divididos em dois grupos: grupo praticante de atividade física (GP) e nãopraticante (GNP). Foram utilizadas a Escala de Avaliação de Equilíbrio e Marcha de Tinetti, Atividades Básicas de Vida Diária e a Avaliação Instrumental de Vida Diária (AIVD) de Lawton. Não houve diferença de idade entre GP (69,4 \pm 7,5 anos) e GNP (71,9 \pm 6,7 anos). Observamos que o GP e GNP apresentaram diferenças estatisticamente significantes nos escores dos domínios de equilíbrio e marcha, bem como no escore total (16 vs 14; 12 vs 11 , 28 vs 24,5, respectivamente). Em relação à Avaliação das Atividades Básicas

Palavras-chave: Idoso. Atividade Motora. Acidentes por Quedas. Atividades Cotidianas. Marcha. Análise Transversal. Estudo Comparativo. Queda. Equilíbrio. Atividade de Vida Diária de Vida Diária, não houve diferença entre os grupos, pois todos os idosos apresentaram pontuação máxima, significando, assim, independência. Apesar de não haver diferença estatisticamente significante no escore de Avaliação Instrumental de Vida Diária (AIVD) entre o GP e GNP (27 vs 27,5, respectivamente), observamos que $70 \%$ dos idosos do GP eram independentes nas atividades diárias e apenas 50\% no GNP, o qual resultou em maior número de idosos necessitando de ajuda para realizar atividades. Concluímos que o GP apresentou melhor equilíbrio e marcha com maior proporção

Universidade Nove de Julho. Curso de Fisioterapia. São Paulo, SP, Brasil

Correspondência / Correspondence

Juliana Assis Magalhães Figliolino

Rua Solidônio Leite, 2718/61, Bl 02 - Vila Ema

03275-000 - São Paulo, SP, Brasil

E-mail: julianaassis_8@hotmail.com 
de idosos com independência nas AIVD e AVD, ou seja, menor probabilidade de queda em relação ao GNP.

\section{Abstract}

The aim of this study was to compare elderly practitioners (GP) and nonpractitioners (GNP) of physical activities in relation to probability of falls, imbalances and difficulties in performing activities of daily living. This is a cross-sectional study with 40 elderly of both genders. The participants were divided in two groups: the practitioners $(\mathrm{PG}, \mathrm{n}=20$ ) and non-practitioners $(\mathrm{NPG}, \mathrm{n}=20$ ) groups. The instruments for evaluation were: Tinetti Gate and Balance Assessment Scale (TGBA scale), Basic Activities of Daily Living Scale (BADL scale) and Lawton Instrumental Activities of Daily Living (LIADL). Age did not differ between PG and NPG $(69.4 \pm 7.5$ yrs vs $71.9 \pm 6.7$ yrs, respectively). There was a significant difference between PG and NPG in the balance and gate scores, and total score as well (16 vs 14; 12 vs 11, 28 vs 24.5, respectively). In relation to BADL scale, no difference was observed between groups because all samples, PG and NPG, presented maximal punctuation, which means independent performance of basic activities of daily living. Despite no significant difference in LIADL scores between PG and NPG (27 vs 27.5 , respectively), we observed that $70 \%$ of elderly in the PG were independent to perform activities of daily living and only $50 \%$ in the NPG, which presented highest number of elderly needing help to perform activities. We concluded that PG showed better balance and gate as compared to NPG, with higher proportion of independent elderly performing instrumental and basic actiivites activities of daily living with less probability of falls.
Key words: Fall. Balance. Walking. Aged. Motor Activity. Accidental Falls. Activities of Daily Living. Gait. Period Analysis. Comparative Study

\section{INTRODUÇÃO}

A maioria dos gerontologistas define o envelhecimento como a redução da capacidade de sobreviver, sendo um processo dinâmico e progressivo no qual há modificações tanto morfológicas quanto funcionais, bioquímicas e psicológicas que determinam progressiva perda da capacidade de adaptação do indivíduo ao meio ambiente, ocasionando maior vulnerabilidade e maior incidência de processos patológicos que terminam por levá-lo à morte. ${ }^{1}$
Com o passar dos anos, o organismo passa por um processo natural de envelhecimento, gerando assim modificações funcionais e estruturais que geram diminuição da vitalidade, favorecendo o aparecimento de doenças, sendo mais prevalentes as alterações sensoriais, doenças ósseas, cardiovasculares e diabetes. $\mathrm{O}$ envelhecimento compromete a habilidade do sistema nervoso central (SNC) em realizar o processamento de sinais vestibulares, visuais e proprioceptivos responsáveis pela manutenção do equilíbrio corporal; esses processos degenerativos são 
responsáveis pela ocorrência de vertigem e/ ou tontura e desequilíbrio. O desequilíbrio é um dos fatores que limitam hoje a vida dos idosos, pois em $80 \%$ dos casos não pode ser atribuído a uma causa específica, mas sim ao um comprometimento do sistema de equilíbrio como um todo. $\mathrm{O}$ controle do equilíbrio requer a manutenção do centro da gravidade sobre a base de sustentação durante situações estáticas e dinâmicas. Cabe ao corpo responder às variações do centro de gravidade, quer de forma voluntária ou involuntária. Este processo ocorre sobretudo de forma eficaz pela ação dos sistemas visual, vestibular e somato-sensorial. . $^{2,3}$ Com o envelhecimento, esses sistemas são afetados e várias etapas do controle postural podem ser suprimidas, diminuindo a capacidade compensatória do sistema, levando a um aumento da instabilidade. ${ }^{4}$

A queda é a consequência mais perigosa do desequilíbrio e da dificuldade de locomoção, sendo seguida por fraturas, deixando os idosos acamados por dias ou meses, e também é responsável por $70 \%$ das mortes acidentais com pessoas maiores de 75 anos. ${ }^{5}$ Ela é definida como um deslocamento não intencional do corpo por um nível inferior à posição inicial, com incapacidade de correção em tempo hábil, podendo ser considerada uma manifestação de patologias existentes que resulta da interação entre fatores ambientais, biomédicos, fisiológicos e psicossociais que compromete a estabilidade do indivíduo, sendo reconhecida como importante problema de saúde pública entre os idosos, em decorrência da frequência, da morbidade e do ele- vado custo social e econômico, devido às lesões provocadas. ${ }^{6} \mathrm{Um}$ dos fatores relacionados ao envelhecimento que pode predispor o indivíduo a quedas é a tendência à lentidão dos mecanismos de integração central, que são importantes para os reflexos posturais. $\mathrm{O}$ envelhecimento parece reduzir a capacidade de processamento e a habilidade de dividir a atenção. Se a concentração for distraída, por exemplo, por outra tarefa cognitiva, há recuperação mais lenta de uma perturbação postural. Aproximadamente $30 \%$ das pessoas de 65 anos ou mais caem pelo menos uma vez por ano. Os principais problemas causados pelas quedas são fraturas, lesões na cabeça, ferimentos graves, ansiedade, depressão e medo de subsequentes quedas. ${ }^{7,8}$

Além de produzirem importante perda de autonomia e de qualidade de vida entre os idosos, as quedas podem repercutir entre seus cuidadores, principalmente os familiares, que devem se mobilizar em torno de cuidados especiais, adaptando toda a rotina em função da recuperação ou adaptação após a queda. ${ }^{4}$

Conforme é citado na literatura, a queda é ocasionada pela diminuição das habilidades de atividade de vida diária, falta de equilíbrio e/ ou déficit de atividade física. O exercício físico pode retardar, melhorar ou prevenir a atividade de vida diária (AVD), equilíbrio e marcha, levando à diminuição do risco de queda.

O objetivo do presente estudo foi comparar idosos que praticam atividades físicas com idosos que não praticam atividade físi- 
ca, em relação à probabilidade de quedas, falta de equilíbrio e dificuldades na realização das AVD.

\section{MATERIAIS E MÉTODOS}

O estudo avaliou a probabilidade de quedas, equilíbrio, marcha e atividades de vida diária em idosos praticantes e não-praticantes de atividade física.

Participaram 40 idosos, de 60 a 90 anos de idade, de ambos os sexos, divididos em dois grupos, sendo o primeiro grupo de 20 idosos que praticavam atividade física e o segundo contendo 20 idosos que não praticavam atividade física; após terem sido selecionados e assinado o termo de consentimento livre e esclarecido.

Os critérios de inclusão foram os seguintes: indivíduos idosos cuja faixa etária era entre 60 e 90 anos de idade; sendo no primeiro grupo somente os praticantes de atividade física como caminhada, hidroginástica/hidroterapia ou exercícios aeróbicos (50 minutos três vezes por semana), e no segundo grupo somente indivíduos sedentários. Ambos os grupos não possuíam qualquer patologia instalada que comprometesse a marcha, mobilidade física e/ou instrumental. Os critérios de exclusão foram: existência de patologias que pudessem interferir na mobilidade física e marcha; indivíduos que faziam uso de medicamentos que interferiam na mobilidade física e/ou equilíbrio e marcha; presença de déficit cognitivo e graves alterações visuais.
Foram aplicados os questionários Escala de Avaliação do Equilíbrio e Marcha de Tinetti ${ }^{9}$; Escala de Atividades Básicas de Vida Diária (Katz); Atividades Instrumentais de Vida Diária (AIVD) de Lawton ${ }^{10}$, a qual foi adaptada para o sexo feminino e para o sexo masculino.

Os indivíduos praticantes de atividade fisica foram selecionados dos grupos de ombro e de fibromialgia pertencentes ao Ambulatório Integrado de Fisioterapia do Centro Universitário Nove de Julho - Unidade Memorial (UNINOVE). Já os não-praticantes de atividade física foram selecionados através de entrevistas com moradores pertencentes ao Condomínio Residencial Vila Belém, no qual residia uma das autoras do presente estudo.

$\mathrm{Na}$ análise estatística, foi utilizado o teste $t$ de Student não-pareado para comparar a idade dos grupos. Os escores resultantes das escalas de Avaliação de Equilíbrio e Marcha de Tinetti, Atividades Básicas de Vida Diária e a Avaliação Instrumental de Vida Diária (AIVD) de Lawton foram comparados em seus valores medianos pelo teste de Mann-Whitney, com um $\mathrm{p}<0,05$ considerado significante. ${ }^{9,10}$

O trabalho apresentado foi aprovado pelo Comitê de Etica em Pesquisa da Universidade.

\section{RESULTADOS}

Ao avaliarmos a amostra total $(\mathrm{n}=40)$, maior número de idosos pertencia ao gê- 
nero feminino $(\mathrm{n}=29,72,5 \%)$. O mesmo foi observado ao analisarmos a distribuição de gêneros entre os grupos, ou seja, foi observado predomínio do gênero feminino tanto em praticantes como em não-praticantes de atividade física ( $65 \%$ vs $80 \%$, respectivamente). Não houve diferença estatisticamente significante na idade entre praticantes e não-praticantes de atividade física
$(69,4 \pm 7,5$ vs $71,9 \pm 6,7$ anos, respectivamente).

Quanto à Escala de Tinetti, ${ }^{9}$ observamos que os praticantes e não-praticantes de atividade física apresentaram diferenças significantes nos escores dos domínios de equilíbrio e marcha, bem como no escore total. Os dados podem ser visualizados na tabela 1 .

Tabela 1 - Comparação dos escores da Escala de Tinetti entre idosos praticantes e não-praticantes de atividade física.

\begin{tabular}{lcc}
\hline Escala de Tinetti & \multicolumn{2}{c}{ Atividade física } \\
\cline { 2 - 3 } & $\begin{array}{c}\text { Praticantes } \\
(\mathrm{n}=20)\end{array}$ & $\begin{array}{c}\text { Não-praticantes } \\
(\mathrm{n}=20)\end{array}$ \\
\hline Escore do equilíbrio & $16(15-16)$ & $14(10-16)^{*}$ \\
Escore da marcha & $12(11-12)$ & $11(9-12)^{*}$ \\
Escore total & $28(26-28)$ & $24,5(20-28)^{*}$ \\
\hline
\end{tabular}

* $\mathrm{p}<0,05$. Valores expressos em mediana (valores mínimos e máximos)

Ao analisarmos a frequência de idosos por escore pontuado, observamos maior proporção de idosos praticantes de atividade física com melhor pontuação em re- lação aos não-praticantes de atividade física, tanto para o domínio equilíbrio (figura 1) quanto para o domínio marcha (figura 2). 


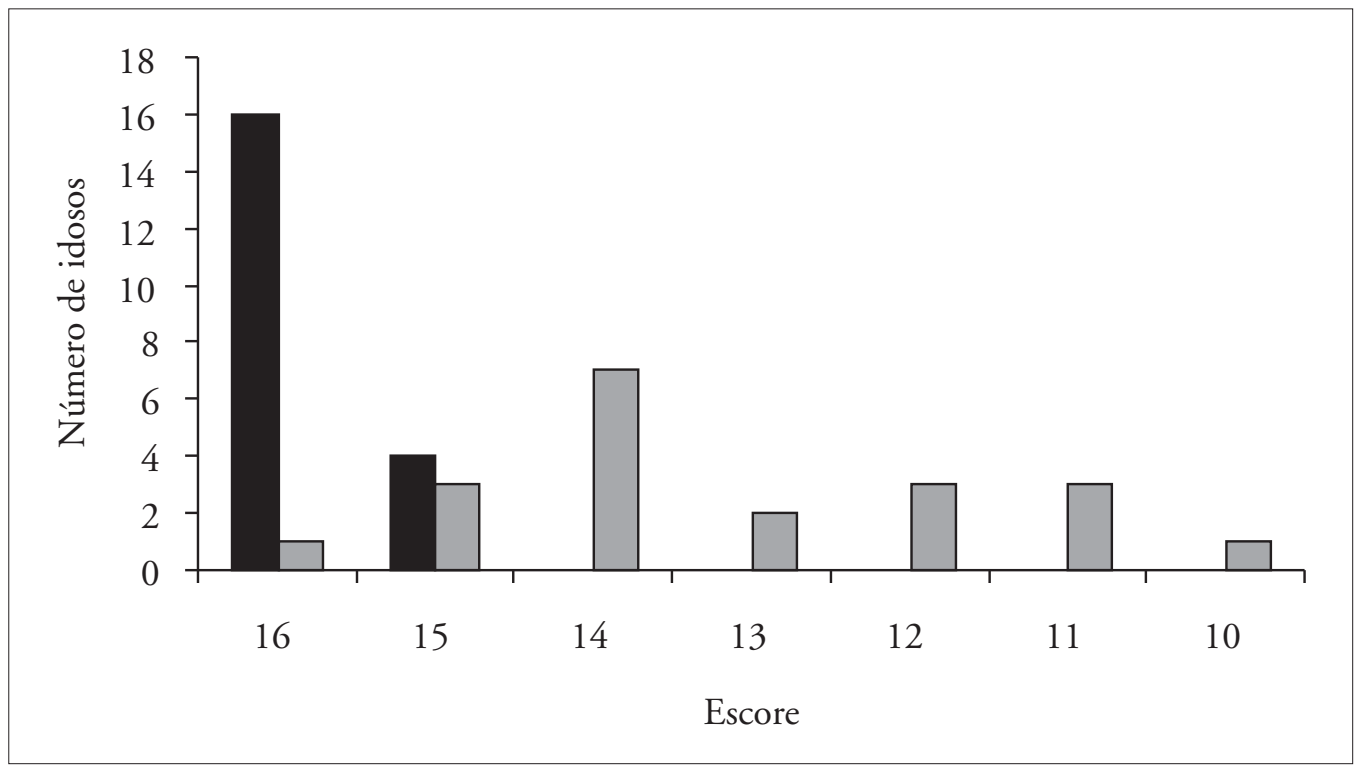

Figura 1 - Frequência de idosos por escore no domínio equilíbrio ( $\square$ P e $\square$ NP).

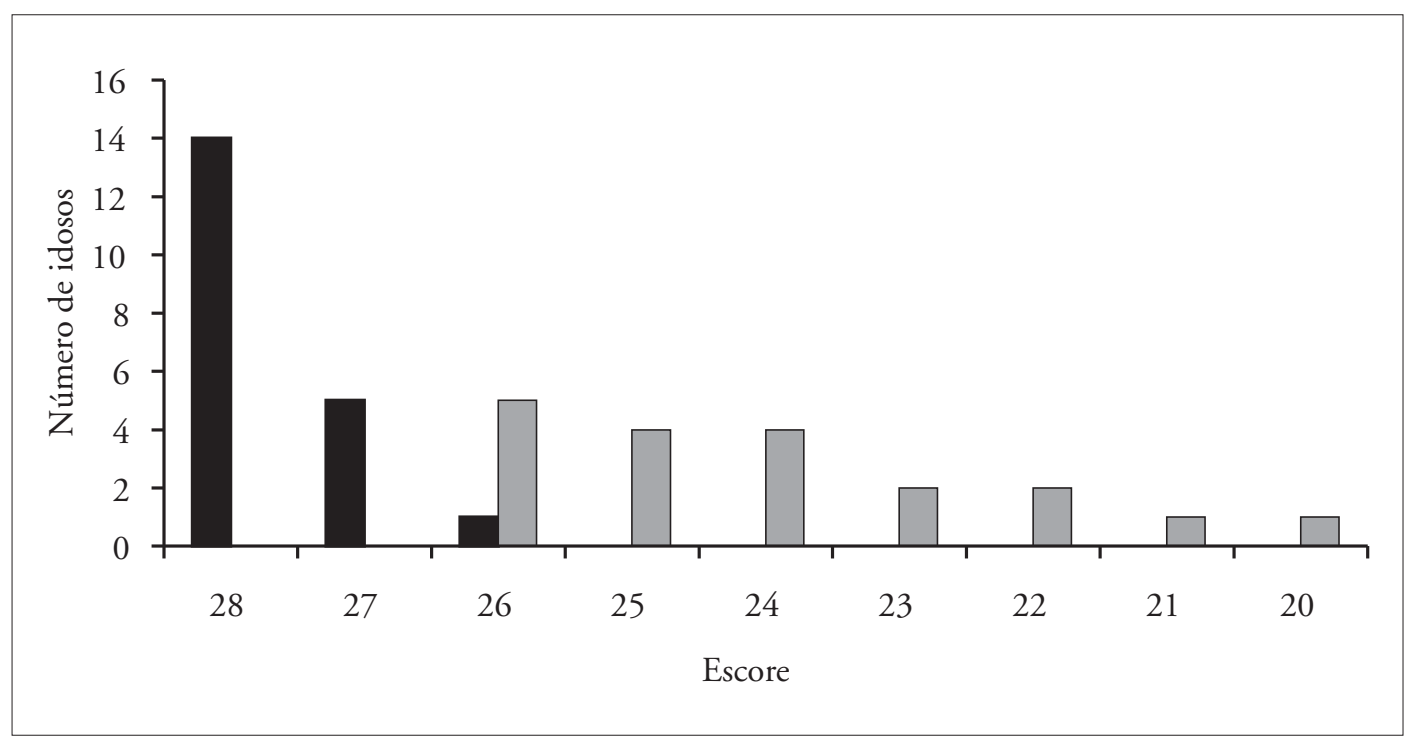

Figura 2 - Frequência de idosos por escore no domínio marcha ( $\square \mathrm{Pe} \quad \mathrm{NP})$. 
Em relação à Avaliação das Atividades Básicas de Vida Diária, não houve diferença entre os grupos, pois todos os idosos apresentaram pontuação máxima nessa escala, o que significa independência para atividades básicas de vida diária, tais como: banho, vestir-se, higiene pessoal, transferência, continência e alimentação.

Quanto à Avaliação Instrumental de Vida Diária (AIVD), não houve diferen- ça estatisticamente significante no escore total entre o GP e GNP (27 vs 27,5, respectivamente). Entretanto, observamos maior número de idosos do GP com escores mais elevados na AIVD, ou seja, a maioria desse grupo era totalmente independente nas suas atividades diárias, enquanto que no GNP apenas a metade do grupo obteve escore total e outra metade necessitava de ajuda para realizar a atividade (figura 3).

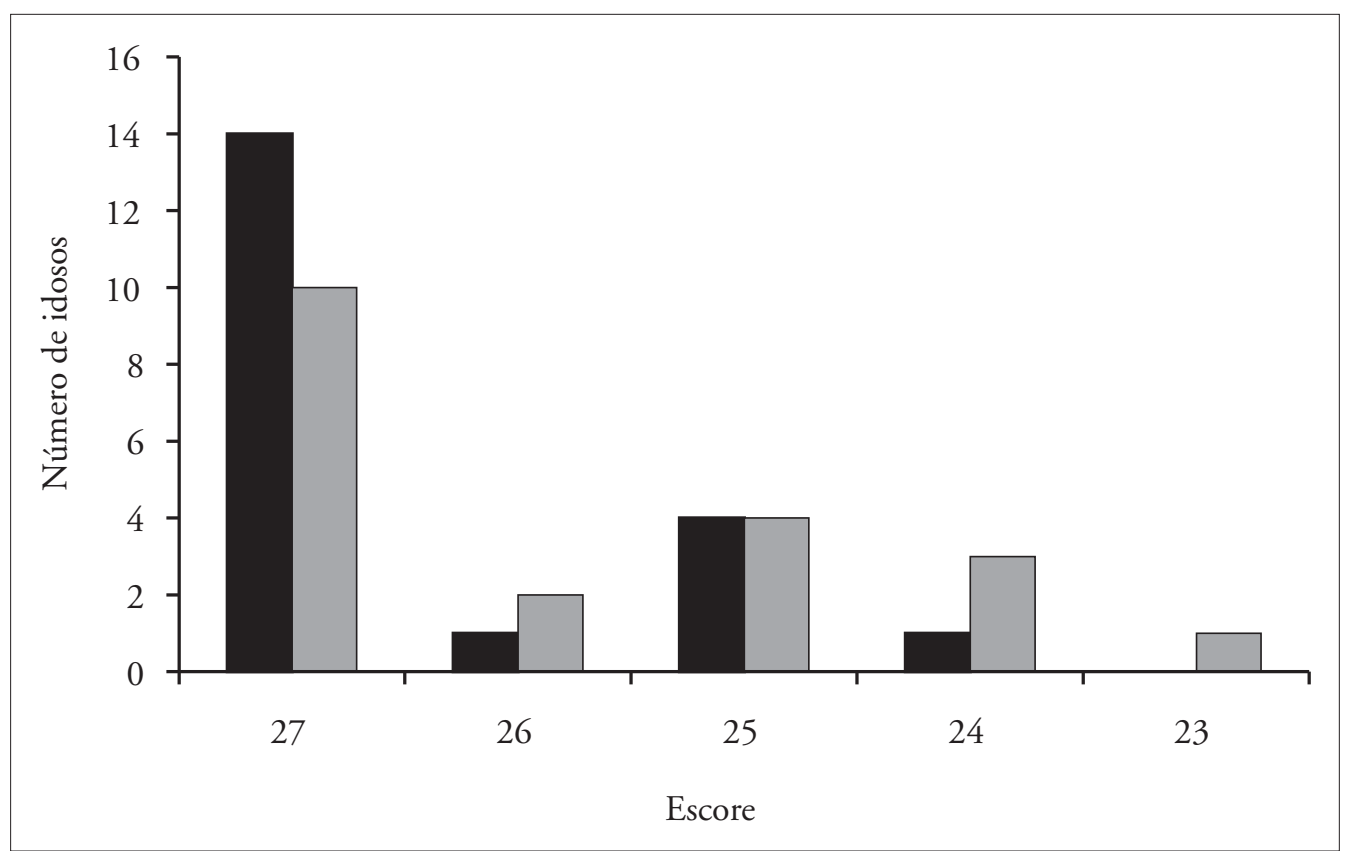

Figura 3 - Frequência de idosos por escore na Avaliação Instrumental de Vida Diária $(\square \mathrm{P}$ e $\square \mathrm{NP})$. 


\section{DISCUSSÃO}

A partir desses achados, foi possível identificar que o grupo não-praticante de atividade física foi mais vulnerável a apresentar alterações na marcha, equilíbrio e nas atividades instrumentais de vida diária.

Quando um indivíduo envelhece, alguns sistemas orgânicos apresentam um declínio de função, sendo comum associá-lo ao processo de envelhecimento, embora muitas dessas alterações possuam poucos efeitos na realização das necessidades diárias da maioria da população idosa. ${ }^{1}$ Apesar de ocorrerem tais alterações nos diferentes sistemas orgânicos dos indivíduos idosos, nos resultados do presente estudo não foi encontrada nenhuma alteração com relação ao escore para avaliação de AIVD, tanto para os praticantes como para os não-praticantes de atividade física.

Segundo Fabrício et al., ${ }^{11}$ a queda traz como consequência para o idoso o aumento de dificuldade e de dependência para realização das atividades de vida diária (AVD), sendo que as mais prejudicadas após a queda foram deitar/levantar-se da cama, caminhar em superfície plana e tomar banho. Relacionando-se ao presente estudo, na análise de AVD não houve dificuldade para realizar as atividades, pois os idosos avaliados não sofreram queda.

Em outro estudo, com um total de 310 idosos residentes em zona urbana do município de Santa Cruz-RN, Maciel \& Guer$\mathrm{ra}^{12}$ avaliaram o equilíbrio dos pacientes atra- vés do uso do Teste de Apoio Unipodal, no qual se pede para o indivíduo se equilibrar em apenas um dos pés com os olhos abertos por no máximo 30 segundos. $\mathrm{O}$ tempo que cada indivíduo conseguia ficar apoiado somente em um dos pés foi medido em três tentativas, e considerou-se a melhor das três; sendo assim, o resultado obtido foi que 167 idosos (53,9\%) foram considerados normais e 143 idosos (46,1\%) não conseguiram fazer apoio unipodal, ficaram menos que 20 minutos apoiados somente em um dos pés ou não realizaram uma dessas posturas. ${ }^{12}$ Comparando esses resultados com os resultados do presente estudo, para a Avaliação de Equilíbrio de Tinetti, dos 20 idosos praticantes de atividade física, $16(80 \%)$ tiveram a pontuação máxima, sendo considerados normais, e dos 20 não-praticantes de atividade física, apenas três (5\%) tiveram a pontuação máxima, sendo que $95 \%$ dos não-praticantes de atividade física tiveram maior propensão a quedas.

Em homens idosos, a velocidade usual e máxima da marcha está fortemente associa$\mathrm{da}$ ao condicionamento físico, e essa associação direta entre a velocidade normal da marcha e condicionamento físico é compatível com a hipótese de que alto nível de atividade física pode manter o condicionamento e a velocidade usual da marcha simultaneamente - ou seja, um estilo de vida ativo que inclua, por exemplo, caminhadas, pode manter uma boa marcha, força muscular e estimular o equilíbrio. As alterações sensoriais também podem influenciar na marcha, principalmente no tocante à acuidade visual e sensibilidade plantar, fatores 
esses também associados às quedas; modificações adicionais relacionadas com o envelhecimento incluem perda do balanço normal dos braços, diminuição da rotação pélvica e do joelho, cadência diminuída e aumento da altura de cada passo - ou seja, os indivíduos mais velhos possuem passos mais curtos, consomem menos tempo em apoio unipodal, caminham com a pelve rodada anteriormente, e quadril ligeiramente fletido e os pés rodados para fora. ${ }^{1}$ Já em outro estudo realizado por Patta et.al., ${ }^{13}$ constatouse que os idosos têm menor capacidade para se adaptar às perturbações adicionais durante a locomoção e, portanto, em ambientes complexos (superfícies irregulares) a locomoção torna-se um grande risco. Comparando-se ao nosso estudo, os idosos que não praticavam atividade física apresentaram maior déficit de equilíbrio, sendo uma das soluções para melhora do equilíbrio a atividade física.

Cozzani \& Castro $^{14}$ constataram que, em geral, as demandas ambientais desafiam a capacidade adaptativa das funções de mobilidade entre idosos, sendo comum que, na presença de obstáculos transponíveis, níveis de força muscular sejam decisivos para eficiência do gesto. Além da força muscular, outros fatores intrínsecos podem levar à escolha de estratégias motoras diferenciadas quando o ambiente é subitamente alterado. Esses autores analisaram a estratégia adaptativa durante $\mathrm{o}$ andar na presença de obstáculos, observando 45 idosos agrupados em três grupos: grupo institucionalizado (GI 13 idosos), grupo ativo (GA 13 idosos) e grupo sedentário (GS 19 idosos). Os resultados desse estudo foram: o GI e GS tiveram estratégias adaptativas iguais, ou seja, diminuíram a velocidade ao chegarem perto de um obstáculo e durante a passada do obstáculo o membro inferior apresentava velocidade maior e mais próxima do obstáculo,sendo que o GA teve melhores estratégias adaptativas, ou seja, a velocidade da marcha era igual perto e longe do obstáculo e durante a passada aproximou-se mais ao comportamento dos indivíduos jovens e com maior distância entre os pés e o obstáculo. Concluiu-se, assim, que indivíduos idosos ativos tinham menor propensão a queda do que indivíduos sedentários. ${ }^{14}$ Comparando esses resultados com o presente estudo, podemos afirmar que em relação à Avaliação da Marcha de Tinetti, ${ }^{9}$ dos idosos que praticam atividade física, $95 \%$ não apresentaram risco de quedas, sendo que apenas $30 \%$ dos idosos não-praticantes de atividade física conseguiram escore total, ou seja, não apresentaram risco de quedas. Conclui-se que $70 \%$ dos idosos não-praticantes de atividade física apresentaram grande risco de quedas.

Os idosos com déficit cognitivo têm cinco vezes mais chances de sofrer quedas. $\mathrm{O}$ controle postural e a manutenção do equilíbrio sofrem influência da função cognitiva, principalmente do sistema atencional. Os idosos que apresentam comprometimento cognitivo associado à alteração de atenção e memória são mais susceptíveis às quedas, por apresentarem as funções protetoras e de julgamento prejudicadas, maior dificuldade para adaptação no ambiente e ado- 
ção de atitudes sem consciência dos riscos que isso representa. ${ }^{1,7,11,15} \mathrm{O}$ presente estudo não analisou a relação entre o déficit cognitivo e quedas, mas sabe-se que esta relação é importante para diagnosticar a probabilidade de queda.

Podsiadlo \& Richardson ${ }^{16}$ avaliaram 60 idosos de um hospital de geriatria com o "Índice de Barthel de ADL", o qual avalia o grau de dependência funcional e cujos resultados foram: 57 idosos eram dependentes para executar atividades básicas da vida diária e somente 3 idosos foram dependentes nas AVD. E também Oliveira, Goretti \& Pereira ${ }^{17}$ analisaram 28 idosos pelo índice de Katz, que avalia a AVD, sendo que $92 \%$ dos pacientes eram totalmente independentes. Comparando esses estudos com o presente, nossos resultados são semelhantes, pois $100 \%$ dos pacientes analisados eram totalmente independente nas AVDs.

Quando o idoso não pratica atividade física, significa que há prejuízo no equilíbrio no que seria o fator primordial no comprometimento das atividades instrumentais, como também limitações da força muscular, da mobilidade e da marcha. Esses idosos são mais vulneráveis a queda, com isso aumenta o risco de morte..$^{18}$ Já em nosso estudo, analisamos as atividades instrumentais onde não houve alterações significantes, mas, por outro lado, ocorreu um grande resultado no déficit de equilíbrio, como descrito acima.

Santos \& Andrade ${ }^{6}$ analisaram a incidência de quedas em idosos institucionalizados na cidade de Salvador-BA, onde foram ob- servados 316 idosos de ambos os sexos no período de abril a dezembro de 2003 . Entre as quedas analisadas, 193 (84,3\%) ocorreram em pessoas que não praticavam nenhum tipo de atividade física, 27 (11,8\%) ocorreram em idosos que realizavam atividade em grupo desenvolvida pelo setor de Fisioterapia, oito $(3,5 \%)$ quedas em idosos que realizam caminhadas e uma $(0,4 \%)$ queda foi sofrida por idoso que fazia tai chi chuan. Em relação ao tempo de início da atividade, dez $(4,4 \%)$ quedas ocorreram em indivíduos que praticavam atividade física há mais de um ano, oito $(3,5 \%)$ quedas em idosos que praticavam atividade entre seis e 12 meses, 12 (5,2\%) quedas em idosos que praticavam atividade entre um e cinco meses, $\mathrm{e}$ seis $(2,6 \%)$ quedas ocorreram em idosos que praticavam atividade a menos de um mês. Concluiu-se, assim, que a maioria dos idosos (ou seja, 84,3\% que sofreram queda) não era praticante de atividade física, o que sugere que a atividade física diminui a propensão a quedas. ${ }^{6}$ Esses autores obtiveram a mesma conclusão que a do presente estudo, ou seja, mostraram que a realização de atividade física regular diminui a probabilidade de quedas.

A prevalência do sedentarismo é elevada em qualquer faixa etária. Atualmente, torna-se preocupante até em crianças, mas dentre os adultos jovens e sobretudo nos idosos, essa prevalência supera a faixa dos $90 \%$. O sedentarismo pode ser responsável por grave estado de limitação da saúde do idoso, podendo caracterizar-se como uma doença potencialmente responsável por importante limitação funcional em idosos cujo 
tratamento específico, com atividade física programada, pode evitar sua instalação, limitar a progressão ou mesmo corrigir as disfunções dela decorrentes. ${ }^{19}$ Com relação ao nosso estudo, observamos que o sedentarismo influencia no equilíbrio dos indivíduos idosos, com isso aumentando o risco de quedas; e que a prática de atividade física pode melhorar a qualidade de vida, diminuindo o risco de quedas.

A atividade física pode ser conceituada para o idoso como "o fenômeno biológico que envolve a contração muscular”. Um programa de atividade física cuidadosamente elaborado constatou a possibilidade de melhora global da condição funcional, incluindo a captação periférica de oxigênio, aumento do HDL, redução dos triglicerídeos, pressão arterial, gordura corporal, devido ao maior gasto calórico, e tendência à elevação da taxa metabólica, pelo aumento de massa muscular. A prática da atividade física vem sendo progressivamente estudada na sua eficácia de reduzir a probabilidade de ocor- rência da maior parte das doenças ou contribuir para a eficácia do seu tratamento. Ela pode interferir na saúde, principalmente quando acompanham de maiores possibilidades de satisfação pessoal e de possibilidades de interação social- sendo assim, a atividade física deve ser estimulada em todas as orientações dirigidas à promoção do envelhecimento saudável. ${ }^{19,20}$ Isso se compara aos resultados obtidos no presente estudo, que mostram a eficácia e as vantagens da prática de atividade física.

\section{CONCLUSÃO}

Diante dos resultados obtidos, podemos observar que os idosos praticantes de atividade física apresentaram melhor equilíbrio, marcha e independência nas AIVD e AVD, ou seja, menor probabilidade de queda; já os indivíduos idosos não-praticantes de atividade física apresentaram como consequência maior propensão a quedas.

\section{REFERÊNCIAS}

1. Carvalho Filho ET, Papaleo Netto M, Garcia YM. Biologia e teorias do envelhecimento. In: Carvalho Filho ET, Papaleo Netto M. Geriatria fundamentos, clínica e terapêutica. São Paulo: Atheneu; 2000. p. 3-18.

2. Romero CA, et al. Actividades preventivas en los ancianos. Gruppos de Expertos Dell Papps. Aten Primaria 2001 nov; 28, (2).
3. Veras RP, Kalache A, Ramos LR. O envelhecimento da população mundial. Um desafio novo. Rev Saúde Pública 1987; 21: $200-10$.

4. Coutinho ESF, Silva SD. Uso de medicamentos como fator de risco para fraturas grave decorrente de quedas em idoso. Cad Saúde Pública 2002 set/out; 18(5): 1359-66. 
5. Ruwer SL, Rossi AG, Simon LF. Equilíbrio no Idoso. Rev Bras Otorrinolaringol 2005; 71(3).

6. Santos MLC, Andrade MC. Incidência de quedas relacionada aos fatores de riscos em idosos institucionalizados. Revista bahiana de saúde pública 2005; 29(1): 57-68.

7. Carvalho A M, Coutinho ESF. Demência como fator de risco para fraturas graves em idosos. Rev Saúde Pública 2002; 36(4).

8. Paixão Júnior CM, Heckmann M. Distúrbios da Postura, Marcha e quedas, In: Freitas EV, et al. Tratado de Geriatria e Gerontologia. Rio de Janeiro: Guanabara Koogan; 2002. p 624-34.

9. Tinetti ME. Performance-oriented assessment of mobility problems in elderly patients. J Am Geriatr Soc 1986 Feb; 34(2): 119-26.

10. Lawton MP, Brody EM. Assessment o folder people: self-maintaining and instrumental activies of daily living. Gerontologist 1969; 9: 179-86.

11. Fabrício SCC, Rodrigues RAP, Costa Junior ML. Causas e consequências de quedas de idosos atendidos em hospital público. Rev Saúde Pública 2004; 38(1): 93-9.

12. Maciel ACC, Guerra RO. Prevalência e fatores associados ao déficit de equilíbrio em idosos. Revista brasileira de ciência \& movimento 2005; 13(1): 37-44.

13. Patta AE, Prentice SD, Gobbi LT. Visual Control of obstacle avoidance during locomotion: strategies in young children, young and older adults. In: Ferrandez A, Teasdale N. Changes in sensory motor behavior in aging. Amsterdam: Elsevier, 1996. p. 257-77.

Recebido: $10 / 3 / 2008$

Revisado: 2/2/2009

Aprovado: 29/5/2009
14. Cozzani M, Mauerberg- Castro EM.

Estratégias adaptativas durante o andar na presença de obstáculos em idosos: impacto da institucionalização e da condição física. Revista Paulista de Educação Física 2005 jan/mar; 19(1): 49-60.

15. Perracini MR, Ramos LR. Fatores associados a quedas em uma coorte de idosos residentes na comunidade. Rev Saúde Pública 2002; 36(6): 709-16.

16. Podsiadlo D, Richardson S. The timed "Up \& Go": a test of basic functional mobility for frail elderly person. J Am Geriatr Soc 1991 fev; 39(2): 142.

17. Oliveira DLC, Goretti LC, Pereira LSM. O desempenho de idosos institucionalizados com alterações cognitivas em atividades de vida diária e mobilidade: estudo piloto. Revista brasileira de fisioterapia 2006; 10(1): 91-6.

18. Ramos LR, Simões EJ, Albert MS. Dependence in activities of daily living and cognitive impaiment strongly predicted mortality in older urban residents in Brazil: a two-year follow-up. J Am Geriatr Soc 2001; 49:1168-75.

19. Jacob Filho W. Atividade física e envelhecimento saudável. In: XI Congresso Ciência do Desporto e Educação Física dos países de língua portuguesa. Revista brasileira de educação física e esporte 2006 set; 20: 73-7.

20. Santarém JM. A importância da atividade física. Disponível em: URL: http:// www.saudetotal.com/artigos/ atividadefisica/idoso.asp. 